\title{
Foundations of Social Knowledge: Editorial Comments
}

\author{
Minoru KARASAWA \\ Nagoya University, Japan
}

How do people acquire knowledge regarding what are the appropriate ways to behave in social settings? How do we come to understand other people's mental states, such as thoughts and feelings? What are the social bases for the construction of selfconcepts? And, at a broader level, how do cultural bases influence our cognition and behavior? These questions concerning foundations of social knowledge have long been pursued by researchers in various fields of psychology, including social, developmental, cognitive, cultural, neuro-scientific, and personality psychologists as well as those from other diverse perspectives. This special issue represents a small and yet highly suggestive sample of such diversity. The article by Lewis, Huang, and Rooksby elucidates the relationship between cognitive skills and social knowledge ("the theory of mind," in particular) among Chinese children, along with potentially complex relationships among other related social and cognitive factors. The study by Wang and Su also examined the theory of mind among Chinese people, but the focus was on adult samples. Using various tasks, these researchers revealed both similarities and differences between adolescents and senior adults. Ito and Takai employed an experimental method to reveal a complex pattern in lay understandings of others' intention that are assumed to operate on their positive versus negative acts. The study by Trnka and Koutnik illustrates that traditional methodologies such as experiments and psychological tests are not the only ways to tap into understandings of other people's mental states. These researchers introduced an innovative research method to the study of recognition of emotional facial expressions. Finally, Ma and Karasawa demonstrate that intergroup contexts as well as cultural background exert significant impact on causal explanations of group members' acts. Taken together, the collection of these unique papers exemplifies pluralistic perspectives in the empirical quest for social bases of human cognition.

Correspondence: Professor Minoru Karasawa, School of Informatics and Sciences, Nagoya University, Furo-cho,Chikusa-ku,Nagoya 464-8601 Japan (e-mail: mkarasawa@nagoya-u.jp). 